\title{
A Group Shuffled BP decoding for punctured Low-Density Parity-Check codes
}

\author{
Sangjoon Park ${ }^{1 \mathrm{a})}$, Sunyoung Lee ${ }^{1}$, Seung-Hoon Hwang ${ }^{2}$, \\ and Sooyong Choi ${ }^{\text {ib) }}$ \\ ${ }^{1}$ School of Electrical and Electronic Engineering, \\ Yonsei University, Seoul 120-749, Korea \\ ${ }^{2}$ Department of Electronics Engineering, \\ Dongguk University-Seoul, 100-715, Korea \\ a)azssa@yonsei.ac.kr \\ b) csyong@yonsei.ac.kr
}

Abstract: A group shuffled belief propagation (GSBP) decoding for punctured low-density parity-check (LDPC) codes is proposed in the paper. The proposed GSBP decoding schedules the updating order of each bit node according to its recovery order in the standard BP decoding. This enables the proposed decoding to recover all punctured nodes in the first iteration with the minimum required number of groups. Calculation of the number of effective updates, which indicates the number of nonzero check-to-bit messages, shows that the proposed decoding can achieve faster decoding convergence than the layered $\mathrm{BP}$ (LBP) decoding for rate-compatible punctured LDPC (RCP-LDPC) codes. In the simulation results, the maximum SNR gain of the proposed decoding over the LBP decoding is about $0.9 \mathrm{~dB}$ at the FER of $10^{-4}$.

Keywords: LDPC codes, GSBP decoding, puncturing, effective update

Classification: Science and engineering for electronics

\section{References}

[1] R. G. Gallager, "Low density parity check codes," MIT Press, 1963.

[2] D. J. C. MacKay, "Good error-correcting codes based on very sparse matrices," IEEE Trans. Inf. Theory, vol. 45, no. 2, pp. 399-431, March 1999.

[3] J. Ha, J. Kim, D. Klinc, and S. W. McLaughlin, "Rate-compatible punctured low-density parity-check codes with short block lengths," IEEE Trans. Inf. Theory, vol. 52, no. 2, pp. 728-738, Feb. 2006.

[4] H. Y. Park, J. W. Kang, K. S. Kim, and K. C. Whang, "Efficient puncturing method for rate-compatible low-density parity-check codes," IEEE Trans. Wireless Commun., vol. 6, pp. 3914-3919, Nov. 2007.

[5] J. Ha, D. Klinc, J. Kwon, and S. W. McLaughlin, "Layered BP decoding for rate-compatible punctured LDPC codes," IEEE Commun. Lett., vol. 11, pp. 440-442, May 2007. 
[6] D. E. Hocevar, "A reduced complexity decoder architecture via layered decoding of LDPC codes," Proc. IEEE SIPS, Dallas, TX, USA, pp. 107112, Dec. 2004.

[7] J. Zhang and M. P. C. Fossorier, "Shuffled iterative decoding," IEEE Trans. Commun., vol. 53, no. 2, pp. 209-213, Feb. 2005.

\section{Introduction}

LDPC codes $[1,2]$ are the techniques in error control coding that enable reliable link connections and efficient processes for both system and mobile services. To adopt these LDPC codes in practical communication systems, rate-adaptability is often requested. Puncturing is one of the major techniques for achieving rate-adaptability at a relatively small implementation complexity cost $[3,4]$. However, the convergence speed of the standard BP decoding [2], the well-known decoding for LDPC codes, decreases as the number of punctured nodes increases $[3,4,5]$.

To accelerate the convergence speed of the standard BP decoding with punctured nodes, the LBP decoding in [5] was proposed for RCP-LDPC codes $[3,4]$. The LBP decoding [6] schedules the updating order of check nodes in a series. Based on this update procedure, the LBP decoding [5] schedules the updating order of check nodes by related punctured nodes at the highest code rate in a system and accelerates the convergence speed for all lower code rates. However, the number of groups for the LBP decoding is larger than the minimum required number of groups to recover all punctured nodes in the first iteration for each lower code rate, and this grows the decoding complexity because of the increased decoding delay $[6,7]$.

In this paper, we propose a GSBP decoding for punctured LDPC codes. The GSBP decoding [7], which is the simplified version of the shuffled BP decoding [7], schedules the updating order of bit nodes in a series. In [7], the updating order of the GSBP decoding is based on a natural increasing order of bit nodes. Instead of natural increasing order, we propose the GSBP decoding for punctured LDPC codes that schedules the updating order of each bit node according to its recovery order in the standard BP decoding. This enables the proposed decoding to recover all punctured nodes in the first iteration to accelerate the convergence speed with the minimum required number of groups, regardless of the number of punctured nodes and the puncturing algorithm. By calculating the number of effective updates, which indicates the number of nonzero check-to-bit messages, and the simulation results, we will show that the proposed decoding can achieve a faster convergence speed than the LBP decoding.

\section{Preliminaries}

Consider an LDPC code of length $N$ and dimension $K$ with an $M(=N-K)$ by $N$ parity check matrix $\mathbf{H}=\left[H_{m n}\right]$, where $H_{m n} \in\{0,1\}$ for $1 \leq m \leq M$, $1 \leq n \leq N$. $M$ and $N$ are the number of check nodes and bit nodes, 
Table I. Scheduling process of the proposed GSBP decoding.

\begin{tabular}{|ll|}
\hline Step 0) & [Initialization] $i:=1, v_{n}:=p_{n}$ for $\forall n \in \mathcal{N}$. \\
Step 1) & {$\left[\right.$ Find and assign $i$-SR nodes] Make a subset $\mathcal{N}_{i}$ of $\mathcal{N}$ such } \\
& that $\forall n \in \mathcal{N}_{i}, v_{n}=1$ and $\sum_{n^{*} \in \mathcal{N}(m)} v_{n^{*}}=1, \exists m \in \mathcal{M}(n)$. \\
Step 2) & [Update parameters] $v_{n}:=0$ for $n \in \mathcal{N}_{i} . i:=i+1$. \\
Step 3) & [Check if there remain punctured nodes to be assigned] \\
& If $\sum_{n \in \mathcal{N}} v_{n} \neq 0$, go to Step 1$)$. \\
Step 4) & {$\left[\right.$ Find and assign 0-SR nodes] Make a subset $\mathcal{N}_{i}$ of $\mathcal{N}$ such } \\
& that $\forall n \in \mathcal{N}_{i}, n \notin \bigcup_{j<i} \mathcal{N}_{j}$.
\end{tabular}

respectively. Let $\mathcal{M}=\{m: 1 \leq m \leq M\}, \mathcal{N}=\{n: 1 \leq n \leq N\}, \mathcal{N}(m)=$ $\left\{n: H_{m n}=1\right\}$, and $\mathcal{M}(n)=\left\{m: H_{m n}=1\right\}$. Assume a punctured LDPC codeword is transmitted, and the number of punctured nodes is $P . r_{\max }$ indicates the highest code rate of the punctured LDPC codewords in a system. The initial log-likelihood ratio (LLR) of a punctured node is set to zero, since a punctured node is not transmitted. Let $\epsilon_{m n}^{i}$ and $z_{m n}^{i}$ be the check-to-bit and bit-to-check messages between the check node $m$ and the bit node $n$ in the $i$ th iteration, respectively.

When the a posteriori LLR of a punctured node $n$ becomes nonzero for the first time in the $i$ th standard BP iteration, a punctured node $n$ is recovered in the $i$ th standard BP iteration, and referred to as the $i$-step-recoverable $(i$ $\mathrm{SR}$ ) node [3]. Unpunctured nodes are referred to as 0-SR nodes. $S R(n)=i$ denotes a $i$-SR node $n$, and $R$ denotes the $\max _{n \in \mathcal{N}} S R(n)$. All check nodes, which recover a punctured node $n$, are called the survived check nodes of $n$.

\section{Proposed decoding}

From $[3,4,5]$, we can observe that $a(>1)$-SR nodes will be recovered in the current decoding stage if all $b(=a-1)$-SR nodes have been previously recovered. In the standard BP decoding, $a$-SR nodes will be recovered after the iteration in which $b$-SR nodes are recovered. However, in the GSBP decoding, if the updating order of $a$-SR nodes follows those of $b$-SR nodes, $a$-SR nodes are recovered in the same iteration in which $b$-SR nodes are recovered. Therefore, we propose a GSBP decoding that assigns $i$-SR nodes to the $i$ th group. This enables the proposed decoding to recover all punctured nodes in the first iteration with the minimum required number of groups, regardless of $P$ and the puncturing algorithm. Generally, since $S R(n)$ for a punctured node $n$ is not known to the decoder, we have to find the $S R(n)$ of each punctured node $n$ in the scheduling. The scheduling process of the proposed decoding is summarized in Table I.

In Table I, $\mathcal{N}_{i}$ represents the $i$ th group of the GSBP decoding. $p_{n}$ is set to 1 if the bit node $n$ is punctured or 0 . Note that the proposed decoding assigns 0-SR nodes to the last scheduling group in order to (1) increase the number of check nodes that the generated nonzero check-to-bit messages to the 0-SR nodes in the first iteration (2) and update punctured nodes firstly 
from the second iteration. ${ }^{1}$

\section{Effective update calculation}

In the LBP decoding proposed in [5] for RCP-LDPC codes [3, 4], assuming the puncturing pattern at $r_{\max }$, survived check nodes of the $l$-SR punctured nodes are assigned to $\mathcal{M}_{l}$, the $l$ th group of the LBP decoding, and the check nodes in which only the 0 -SR nodes participate are assigned to $\mathcal{M}_{R+1}$. This allows the LBP decoding to recover all punctured nodes in the first iteration for all code rates using one scheduling while the decoding complexity for the lower code rates grows larger because of the increased decoding delay [6, 7].

In this section, we will compare the convergence speed of the proposed decoding and the LBP decoding with respect to the number of effective updates when the code rate is $r_{\max }$, which indicates both decodings operate with the minimum required number of groups to recover all punctured nodes in the first iteration. An effective update indicates a nonzero check-to-bit message, $\epsilon_{m n}^{i}$. Let $e^{i}(\mathcal{A})$ be the number of nonzero $\epsilon_{m n}^{i}$ in the $i$ th iteration where $m$ or $n$ is included in set $\mathcal{A}$. Then $e^{i}\left(\bigcup_{\forall s} \mathcal{N}_{s}\right)$ and $e^{i}\left(\bigcup_{\forall l} \mathcal{M}_{l}\right)$ represent the number of effective updates of the proposed decoding and the LBP decoding in the $i$ th iteration, respectively. Since no unrecovered punctured nodes exist after the first iteration for both decodings, $e^{i}\left(\bigcup_{\forall s} \mathcal{N}_{s}\right)=e^{i}\left(\bigcup_{\forall l} \mathcal{M}_{l}\right)$ if $i>1$. In addition, the recovery process of the punctured nodes is the same in both decodings, $e^{1}\left(\bigcup_{\forall s \neq R+1} \mathcal{N}_{s}\right)=e^{1}\left(\bigcup_{\forall l \neq R+1} \mathcal{M}_{l}\right)$. Consequently,

$$
\sum_{i=1}^{I_{\text {max }}} e^{i}\left(\bigcup_{\forall s} \mathcal{N}_{s}\right)-\sum_{i=1}^{I_{\max }} e^{i}\left(\bigcup_{\forall l} \mathcal{M}_{l}\right)=e^{1}\left(\mathcal{N}_{R+1}\right)-e^{1}\left(\mathcal{M}_{R+1}\right),
$$

where $I_{\max }$ is the maximum number of iterations, and $e^{1}\left(\mathcal{N}_{R+1}\right)$ and $e^{1}$ $\left(\mathcal{M}_{R+1}\right)$ are the number of effective updates of the proposed decoding and the LBP decoding for the 0-SR nodes in the first iteration, respectively.

Since no punctured nodes participated in the check nodes in $\mathcal{M}_{R+1}$, $e^{1}\left(\mathcal{M}_{R+1}\right)$ is calculated by the summation of the degree of each check node in $\mathcal{M}_{R+1}$ as follows:

$$
e^{1}\left(\mathcal{M}_{R+1}\right)=\sum_{m \in \mathcal{M}_{R+1}}|\mathcal{N}(m)|
$$

For $e^{1}\left(\mathcal{N}_{R+1}\right), e^{1}\left(\mathcal{M}_{R+1}\right)$ is included in $e^{1}\left(\mathcal{N}_{R+1}\right)$ since $\mathcal{N}_{R+1}$ is a set of the 0 -SR nodes and every check node $m$ that should be assigned in $\mathcal{M}_{R+1}$ in the LBP decoding has only the 0-SR nodes as its neighbors. Moreover, the 0-SR node $n$ also receives the nonzero $\epsilon_{m n}^{1}$ from its neighbor $m$ if all $n^{\prime} \in \mathcal{N}(m) \backslash n$ have nonzero $z_{m n^{\prime}}^{1} . \quad z_{m n^{\prime}}^{1}$ becomes nonzero if $n^{\prime}$ is an 0-SR node or $n^{\prime}$ is punctured but has survived check nodes in $\mathcal{M}\left(n^{\prime}\right) \backslash m$. Let $\mathcal{V}$ be the set of the survived check nodes such that all bit nodes $n^{\prime}(\in \mathcal{N}(m)$ where $m \in \mathcal{V}$ ) have nonzero $z_{m n^{\prime}}^{1}$ after the recovery of all punctured nodes. Then

\footnotetext{
${ }^{1}$ The bit-to-check messages of the punctured nodes are quite smaller than those of the 0 -SR nodes even after the punctured nodes are recovered.
} 


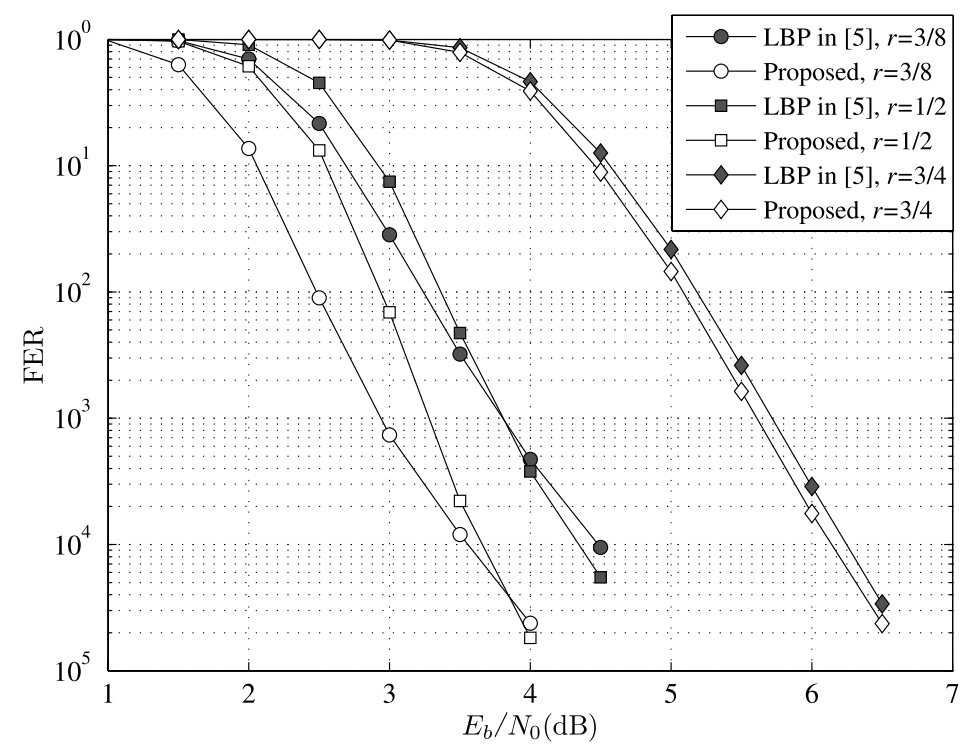

Fig. 1. FERs of the proposed decoding and the LBP decoding.

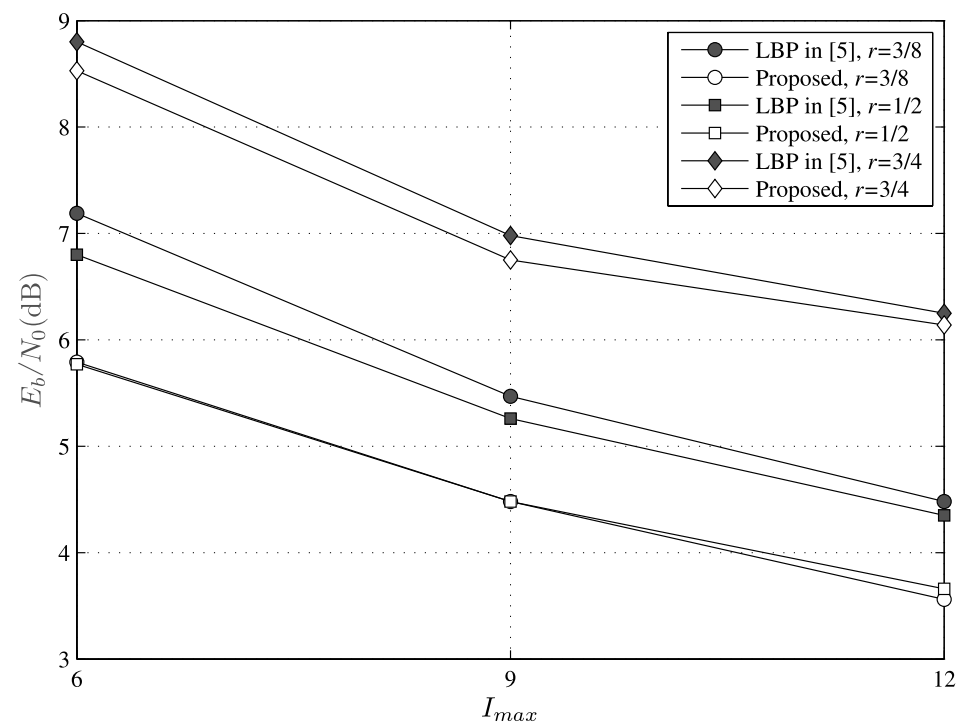

Fig. 2. The required $E_{b} / N_{0}$ values for the proposed decoding and the LBP decoding at the FER of $10^{-4}$.

$e^{1}\left(\mathcal{N}_{R+1}\right)$ is given by

$$
e^{1}\left(\mathcal{N}_{R+1}\right)=e^{1}\left(\mathcal{M}_{R+1}\right)+\sum_{m \in \mathcal{V}}\left(|\mathcal{N}(m)|-\sum_{n \in \mathcal{N}(m)} p_{n}\right) .
$$

\section{Simulation results}

We consider a mother code with a code rate of $r=1 / 4$ and $N=2304$, where the degree distributions of bit nodes and check nodes are $\lambda(x)=0.3546 x+$ $0.2107 x^{2}+0.0937 x^{4}+0.0952 x^{5}+0.2458 x^{13}$ and $\rho(x)=0.4963 x^{3}+0.5021 x^{4}+$ $0.0016 x^{5}$, respectively. $I_{\max }=12$, and AWGN channel and BPSK modulation are assumed. The mother code is punctured using the algorithm in [4]. 
In Fig. 1, we compared the average FER of the proposed decoding and the LBP decoding when each $r$ is considered as $r_{\text {max }}$. The numbers of each group are 2,6 , and 11 when $r=3 / 8,1 / 2$, and $3 / 4$, respectively. $e^{1}\left(\mathcal{N}_{R+1}\right)-e^{1}\left(\mathcal{M}_{R+1}\right)$ are 4920,2463 , and 179 when $r=3 / 8,1 / 2$, and $3 / 4$, respectively. The proposed decoding shows the better performances than the LBP decoding, and the performance gain is maximized at $r=3 / 8$, which has the largest $e^{1}\left(\mathcal{N}_{R+1}\right)-e^{1}\left(\mathcal{M}_{R+1}\right)$. The SNR gains at the FER of $10^{-4}$ are about $0.9 \mathrm{~dB}, 0.7 \mathrm{~dB}$, and $0.1 \mathrm{~dB}$ when $r=3 / 8,1 / 2$, and $3 / 4$, respectively.

Fig. 2 shows the required $E_{b} / N_{0}$ values for each decoding method in Fig. 1 at the FER of $10^{-4}$ with various $I_{\max }$. The performance gain of the proposed decoding over the LBP decoding is more significant with the smaller $I_{\max }$. This can also be observed from the results in Section 4 , since $e^{i}\left(\bigcup_{\forall s} \mathcal{N}_{s}\right)=$ $e^{i}\left(\bigcup_{\forall l} \mathcal{M}_{l}\right)$ when $i>1$. Also, regardless of $I_{\max }$, the performance gain of the proposed decoding over the LBP decoding is maximized at $r=3 / 8$, which has the largest $e^{1}\left(\mathcal{N}_{R+1}\right)-e^{1}\left(\mathcal{M}_{R+1}\right)$.

\section{Conclusion}

In this paper, we proposed a GSBP decoding for punctured LDPC codes. The proposed decoding recovers all punctured nodes in the first iteration to accelerate the decoding convergence with the minimum required number of groups, regardless of $P$ and the puncturing algorithm. Calculation of the number of effective updates shows that the proposed decoding can achieve faster decoding convergence than the LBP decoding. The simulation results also verify the effectiveness of the proposed decoding over the LBP decoding. 